\title{
CORRIGENDUM
}

\section{Association of LILRA2 (ILT1, LIR7) splice site polymorphism with systemic lupus erythematosus and microscopic polyangiitis}

K Mamegano, K Kuroki, R Miyashita, M Kusaoi, S Kobayashi, K Matsuta, K Maenaka, M Colonna, S Ozaki, H Hashimoto, Y Takasaki, K Tokunaga and N Tsuchiya

Genes and Immunity (2008) 9, 650; doi:10.1038/gene.2008.71

Correction to: Genes and Immunity (2008) 9, 214-223; doi:10.1038/gene.2008.5

Since the publication of this paper, the authors have noticed that the legend to the Figure 2 was incorrect;
'G-allele' should read 'A-allele'. The correct legend is shown below.

The authors would like to apologize for this error.

a

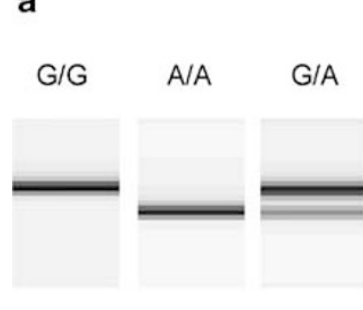

C

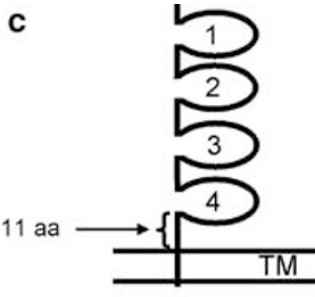

G allele

common form

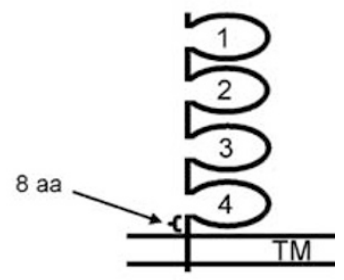

A allele $\triangle 419-421$

b

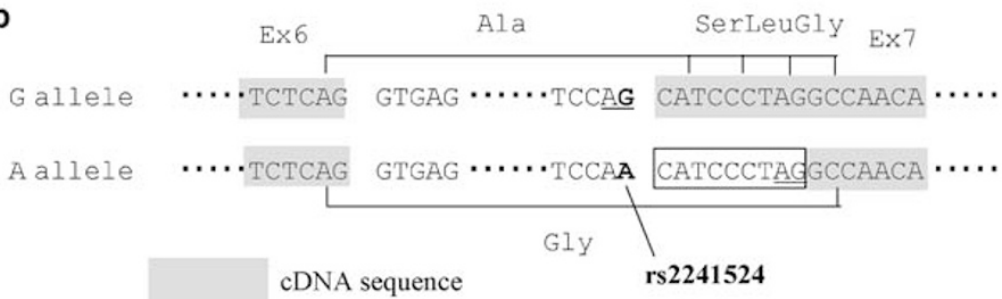

Figure 2 LILRA2 splice acceptor site single nucleotide polymorphism (SNP) rs2241524 activates a cryptic splice acceptor site and causes inframe deletion of three amino acids in the linker region. (a) Capillary electrophoresis of reverse transcriptase (RT)-PCR products from individuals with each genotype revealed two fragments of different sizes that were completely segregated according to the genotype. (b) DNA sequencing revealed that A-allele transcript employs a cryptic splice acceptor site. This results in the deletion of 9 nucleotides (9-nt) (open box) that leads to in-frame deletion of three amino acids. The splice acceptor site used by each allele is shown by underline. (c) The deletion of three amino acids in the LILRA2 protein product from A-allele leads to shortening of the linker region. 